\title{
Solid Pseudopapillary Neoplasm of the Pancreas in a 15-Year-Old Female: A Case Report
}

\section{Mohd Raashid Sheikh ${ }^{\star}$ and Mohammad Taha Syed}

390 E oakenwald St, Dallas, Texas 75203, USA

"Corresponding author: Mohd Raashid Sheikh, 390 E oakenwald St, Dallas, Texas 75203, USA, Tel: 001310-948-2216; Fax: 323-442-9077; E-mail: smraashid@gmail.com

Received date: March 24, 2015, Accepted date: May 5, 2015, Published date: May 11, 2015

Copyright: @ 2015 Raashid SM et al. This is an open-access article distributed under the terms of the Creative Commons Attribution License, which permits unrestricted use, distribution, and reproduction in any medium, provided the original author and source are credited.

\section{Abstract}

Solid pseudopapillary tumor (SPT), also known as Frantz tumor is an unusual form of pancreatic neoplasm. Its natural history differs from the more common pancreatic adenocarcinoma in that it has a female predilection, is more indolent, and carries a better prognosis. Metastatic disease can occur, usually involving the liver, and its management is not well defined.

\section{Case Report}

A 15 year old female presented with vague left sided abdominal pain over 24 hours in duration. The patient had no significant past medical history. Physical exam was significant for mild epigastric tenderness to palpation, but was otherwise unremarkable. Routine lab work was obtained showing normal amylase, AST/ALT, total bilirubin, and a mildly elevated alkaline phosphatase at 172. Abdominal ultrasound was obtained showing a $5 \times 6.3 \times 5.6 \mathrm{~cm}$ mass in the epigastrium. Follow up CT scan revealed a high density cystic mass at the hepatic hilum concerning for choledochal cyst, duplication cyst, or cystic pancreatic neoplasm (Figure 1).

MRCP was obtained to further delineate the masses relationship to biliary tree. The MRCP was concerning for a heterogeneous solid tumor, a possible lymphoma, neuroendocrine tumor or SPT of pancreas (Figure 2).

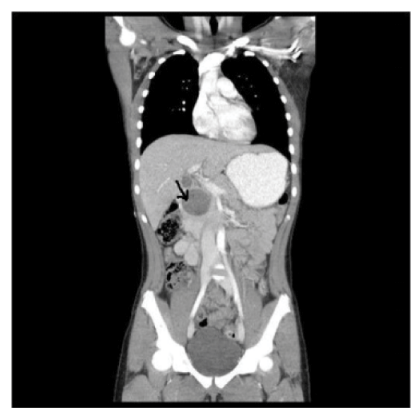

Figure 1: CT Chest/Abdomen/Pelvis: Coronal Image.

CT guided biopsy was performed in an attempt to obtain a pathological diagnosis. Final pathology revealed an epithelial neoplasm, most consistent with SPT of the pancreas.

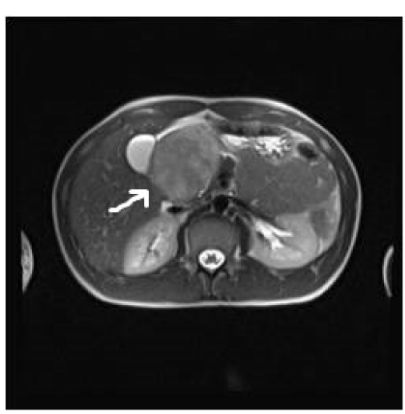

Figure 2: MRCP: Cross Sectional Image.

On exploration a large, well circumscribed, heterogeneous lesion was present in the head of pancreas. Standard pancreaticoduodenectomy was performed (Figure 3).

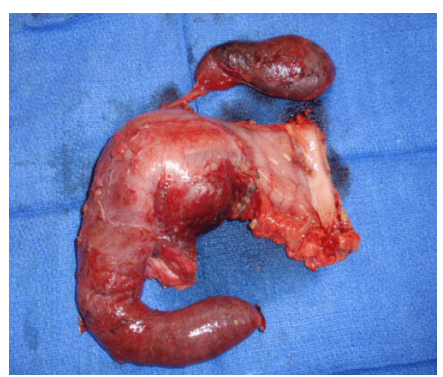

Figure 3: Gross Standard Pancreaticoduodenectomy Specimen.

Final pathology of surgical specimen was SPT of the pancreas (5.1 $\mathrm{cm}$ ) with extensive hemorrhage (Figure 4). Immunohistochemical stains were performed. The tumor cells were CD10 positive, BetaCatenin positive, progesterone receptor positive and chromogranin negative. 


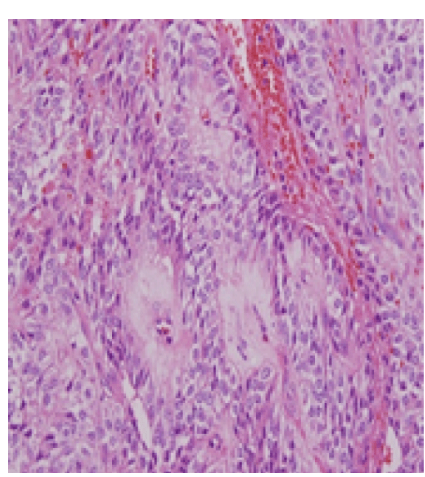

Figure 4: Pathologic Specimen showing Pseudopapillae with hyalinized fibro vascular cores.

\section{Discussion}

SPT of the pancreas was first described by Dr. Frantz in 1959 [1]. It is a very rare tumor accounting for $1 \%$ of all pancreatic exocrine tumors [2]. Due to its rare nature, its histogenesis is still unclear [3]. SPT have a gender predilection for females $(>80 \%)$ and occur in the third and fourth decades of life [4]. Pancreatic SPT is often solitary. It is of low malignant potential, but has been shown to produce extensive local invasion [2]. Extrapancreatic SPT can occur in the retroperitoneum, mesentery, liver, great omentum, ovary, duodenum, stomach, or lung $[5,6]$. SPT can undergo malignant changes as it grows, and such changes occur in $10 \%-15 \%$ of cases. The liver is the most common organ affected by metastasis [7-9].

On histology, SPT appears as an encapsulated tumor composed of a mixture of cystic, solid, and hemorrhagic components. Degenerative changes lead to extensive fibrosis, hemorrhage, focal calcification, and occasional ossification. The doubling time appears to be very slow at 765 days [10]. Malignancy is based on histopathologic findings of vascular and perineural infiltration [10]. The high proliferative index assessed by immunohistochemical staining for Ki-67 may predict poor outcome of malignant SPT [11]. Immunohistochemical characteristics include Loss of membrane expression of $\beta$-catenin and E-cadherin with nuclear positivity can be used confirm the diagnosis of SPT. These tumors are chromogranin negative. Notoharra et al have reported that CD10, CD56, vimentin, synaptophysin, chromogranin, and Pan CK are useful in distinguishing between SPTs and NETs $[12,13]$. SPTs are genetically characterized by activation of betacatenin and its target genes in the WNT signaling pathway [14].

US is the screening imaging method that is usually performed on children because of its wide accessibility and absence of radiation. On US, SPT is a well-circumscribed mass surrounded by a pseudocapsule of compressed pancreatic tissues and reactive fibroses [15].

Abdominal CT scan or MRI is then usually required for locoregional and distant staging. Native CT may identify hemorrhage and calcifications. Calcification is detected in almost $30 \%$ of published cases of adult patients. CECT reveals an enhancement of both tumor pseudocapsule and solid tumor components. MRI should be considered the best imaging technique for children due to the absence of radiation and its improved capacity for visualizing tumor components, especially intratumoral areas of hemorrhage. Both the fibrous capsule and the intramural hemorrhage are important clues for the final diagnosis because they are rarely found in other pancreatic neoplasms [16].

EUS-FNA could be used to increase the accuracy of diagnosis. However, using EUS-FNA to biopsy the pancreas in patients with suspected SPT may be contraindicated because the procedure lacks utility and may increase the possibility of peritoneal seeding [17].

Recommended treatment is radical resection. Surgical resection is usually possible and curative. Therefore, complete aggressive resection is the treatment of choice for SPT even in presence of metastases. Resection of distant metastases should be done at the time of primary resection. Resection should also be tried for recurrences. Extensive lymphadenectomy is not necessary. When successful, 5-year survival is 95\%; when incomplete, median survival rate is 5.7 years [3]. Recurrence is found to occur in about $6 \%$ of patients. The most common sites of recurrence being the liver and the lung $[3,18]$.

Laparoscopic pancreatic resection is a safe and feasible procedure that could become the treatment of choice for patients affected by pancreatic SPT [2]. In the case of incomplete resection, some therapeutic options have been investigated including chemotherapy, radiotherapy, and chemoembolization but are still under evaluation.

In conclusion, SPT of the pancreas is a rare neoplasm, but its incidence is increasing. Adequate operative resection, including laparoscopic surgery, is the mainstay of treatment.

\section{References}

1. Frantz VK (1959) Atlas of tumor pathology. Tumors of the pancreas. Armed Forces Institute of Pathology.

2. Campanile M, Nicolas A, LeBel S, Delarue A, Guys JM, et al. (2011) Frantz's tumor: is mutilating surgery always justified in young patients? Surg Oncol 20: 121-125.

3. Papavramidis T, Papavramidis S (2005) Solid pseudopapillary tumors of the pancreas: review of 718 patients reported in English literature. J Am Coll Surg 200: 965-972.

4. Butte JM (2011) Solid pseudopapillary tumors of the pancreas. Clinical features, surgical outcomes, and long-term survival in 45 consecutive patients from a single center. J Gastrointest Surg 15: 350-7.

5. Takahashi Y, Fukusato T, Aita K, Toida S, Fukushima J, et al. (2005) Solid pseudopapillary tumor of the pancreas with metastases to the lung and liver. Pathol Int 55: 792-796.

6. Zhu H, Xia D, Wang B, Meng H (2013) Extrapancreatic solid pseudopapillary neoplasm: Report of a case of primary retroperitoneal origin and review of the literature. Oncol Lett 5: 1501-1504.

7. Chung YE, Kim MJ, Choi JY, Lim JS, Hong HS, et al. (2009) Differentiation of benign and malignant solid pseudopapillary neoplasms of the pancreas. J Comput Assist Tomogr 33: 689-694.

8. Sperti C, Berselli M, Pasquali C, Pastorelli D, Pedrazzoli S (2008) Aggressive behaviour of solid-pseudopapillary tumor of the pancreas in adults: a case report and review of the literature. World J Gastroenterol 14: 960-965.

9. Kloppel G (2000) Solid-pseudopapillary neoplasm, in World Health Organization classification of tumours: pathology and genetics of tumours of the digestive system. IARC Press: Lyons 246-248.

10. Kato T, Egawa N, Kamisawa T, Tu Y, Sanaka M, et al. (2002) A case of solid pseudopapillary neoplasm of the pancreas and tumor doubling time. Pancreatology 2: 495-498.

11. Tipton SG, Smyrk TC, Sarr MG, Thompson GB (2006) Malignant potential of solid pseudopapillary neoplasm of the pancreas. Br J Surg 93: 733-737. 
Citation: Raashid SM, MS Taha (2015) Solid Pseudopapillary Neoplasm of the Pancreas in a 15-Year-Old Female: A Case Report. Trop Med Surg 3: 185. doi:10.4172/2329-9088.1000185

Page 3 of 3

12. Notohara K, Hamazaki S, Tsukayama C, Nakamoto S, Kawabata K, et al. (2000) Solid-pseudopapillary tumor of the pancreas: immunohistochemical localization of neuroendocrine markers and CD10. Am J Surg Pathol 24: 1361-1371.

13. Uppin SG (2015) Solid-pseudopapillary neoplasm of the pancreas: A clinicopathological and immunohistochemical study of 33 cases from a single institution in Southern India. Indian J Pathol Microbiol 58: 163-9.

14. Abraham SC (2002) Solid-pseudopapillary tumors of the pancreas are genetically distinct from pancreatic ductal adenocarcinomas and almost always harbor beta-catenin mutations. Am J Pathol 160: 1361-9.

15. Buetow PC, Buck JL, Pantongrag-Brown L, Beck KG, Ros PR, et al (1996) Solid and papillary epithelial neoplasm of the pancreas: imagingpathologic correlation on 56 cases. Radiology 199: 707-711.
16. Al-Qahtani S, Gudinchet F, Laswed T, Schnyder P, Schmidt S, et al. (2010) Solid pseudopapillary tumor of the pancreas in children: typical radiological findings and pathological correlation. Clin Imaging 34: 152-156.

17. Jani N, Dewitt J, Eloubeidi M, Varadarajulu S, Appalaneni V, et al. (2008) Endoscopic ultrasound-guided fine-needle aspiration for diagnosis of solid pseudopapillary tumors of the pancreas: a multicenter experience. Endoscopy 40: 200-203.

18. El Nakeeb A, Abdel Wahab M, Elkashef WF, Azer M, Kandil T (2013) Solid pseudopapillary tumour of the pancreas: Incidence, prognosis and outcome of surgery (single center experience). Int J Surg 11: 447-457. 